\title{
Überlegungen zur Prosodie im Bereich DaF ${ }^{1}$
}

\author{
Manuela Moroni, Heinrich Graffmann, Klaus Vorderwülbecke
}

\begin{abstract}
Zusammenfassung
Neben kurzen Bestandsaufnahmen vom Status der Prosodie in Grammatiken und in DaFDidaktiken und -Lehrwerken wird Prosodie näher bestimmt und ihre wichtigsten Eigenschaften und Funktionen in Wort, Ausspruch und Gespräch beschrieben. Im Weiteren wird vor allem die bedeutungsgestaltende Funktion der Prosodie herausgearbeitet. Aus phonologischer Sicht sehen wir die Informationsstruktur als zentral für die Vermittlung der Prosodie an. Anhand von Akzentgruppe und Intonationsphrase wird ihre Rolle bei der rhythmischen Gliederung von Aussprüchen vorgestellt. Als weiteres Beispiel für die kommunikative Funktion von Prosodie wird ihre Rolle beim Ausdruck von Emotion behandelt.
\end{abstract}

\section{Einleitung}

Das Material der Prosodie ist die gesprochene Sprache. Im Gegensatz zur geschriebenen Sprache, deren Beschreibung eine lange Tradition hat, sind empirische Untersuchungen über die gesprochene Sprache erst vor ca. 40 Jahren in Gang gekommen und keineswegs allgemein bekannt. Dieser Beitrag bilanziert zunächst, wieweit gesprochene Sprache und Prosodie in Grammatiken sowie Didaktiken und Lehrwerken DaF Eingang gefunden haben.

Nach begrifflichen Klärungen von Prosodie und Beschreibungen ihrer Funktion auf verschiedenen Ebenen des Sprachsystems folgen Ausführungen zu den Themenbereichen Informationsstruktur, rhythmische Einheiten und Ausdruck von Emotion. Aus phonologischer Perspektive wird immer auch die bedeutungsgestaltende Funktion der prosodischen Mittel herausgearbeitet. Dabei wird auch in eigenen Abschnitten und in eingekästelten Passagen die didaktischmethodische Relevanz der beschriebenen Erscheinungen mit z. T. praktischen Anregungen für den Unterricht aufgezeigt.

\section{Prosodie in Grammatiken sowie Di- daktiken und Lehrwerken DaF}

\section{Prosodie in Grammatiken}

Die Erforschung der gesprochenen Sprache oder genauer: die Wahrnehmung der gesprochenen Sprache als eigenständi-

1 Wir haben diesen Aufsatz gemeinsam konzipiert und verantworten ihn auch gemeinsam. Die einzelnen (Unter)kapitel wurden wie folgt bearbeitet: MM: 2; $4.3 \mathrm{HG:} \mathrm{Abschnitt}$ »Prosodie im Ausspruch « in 2; 3; 5.1. KV: 2; 4.1+2; 5.2. Einleitung und Kap. 6 haben wir gemeinsam geschrieben. 
gen Forschungsgegenstands in der Linguistik ist die Voraussetzung dafür, dass sich Erforschung und Didaktisierung von Prosodie im Bereich DaF entwickeln konnte (siehe Kapitel 2).

Für die Überlegungen in diesem Aufsatz ist vor allem von Bedeutung, dass der Gegenstand gesprochene Sprache und damit auch die prosodischen Gegebenheiten des Deutschen systematisch Eingang in einige neuere Grammatiken gefunden haben. (Zur Berücksichtigung von Gesprochener Sprache in früheren Grammatiken siehe Vorderwülbecke 2006: 567 ff.).

Die Grammatik der deutschen Sprache (Zifonun/Hoffman/Strecker 1997) erhebt den Anspruch, die gesprochene Sprache angemessen $\mathrm{zu}$ berücksichtigen. So wird z. B. die Intonation als grammatisches Mittel betrachtet und bei der Bestimmung der Satztypen mit einbezogen. Der auffälligste Unterschied zu allen vorangegangenen Grammatiken ist die explizite Beschreibung der gesprochenen Sprache im Kapitel »Diskurs und Mündlichkeit «, in dem alle wichtigen prosodischen Mittel ausführlich behandelt werden wie Töne und Tonmuster, Wortakzent und Gewichtungsakzent sowie Pausen und Grenzsignale.

Die 7. Auflage der Duden-Grammatik von 2005 enthält neben einem Kapitel zu »Phonem und Graphem « eins zur »Intonation « (von Jörg Peters) sowie als große Neuerung ein Kapitel zur gesprochenen Sprache (von Reinhard Fiehler).

Im Kapitel »Gesprochene Sprache« werden fast alle prosodischen Mittel kurz beschrieben, für deren Vermittlung im FU wir plädieren: Intonationsphrasen, Tonhöhenverlauf, Pausen, Akzente, Sprechgeschwindigkeit und Lautstärke (Duden 2005: 1206). Es wird aber auch eine Warnung ausgesprochen, die für die Sprachvermittlung berücksichtigt werden muss:
»Die kommunikative Funktion der prosodischen Gestaltungsmittel ist in der Regel nicht eindeutig, sondern mehrdeutig und muss im jeweiligen Kontext erschlossen werden.« (Duden 2005: 1206)

Deshalb plädieren wir auch in diesem Beitrag dafür, dass die kommunikative Situation und damit der Sprecher-HörerBezug stärker in die Arbeit an der Prosodie einbezogen werden.

\section{Prosodie in Didaktiken DaF}

Rösler (1994) widmet »Aussprache und Intonation « zweieinviertel Seiten, davon eine dreiviertel Seite der Intonation (entspricht zum großen Teil der Prosodie bei uns). Er übernimmt die Kritik, dass sich Lehrwerke oft nur auf Übungen zum Phoneminventar beschränken und die Intonation häufig ausblenden (Rösler 1994: 46). Zur Bedeutung der prosodischen Mittel sagt er:

»Rhythmus und Intonation bestimmen die Gliederung und Verständlichkeit des Gesagten und übermitteln Untertöne, die eine Äußerung als sachlich, erregt, höflich oder erstaunt markieren. Notwendig sind deshalb schon früh der Einbezug der emotionalen Ebene und die Einbeziehung der Kommunikationssituation. (Rösler 1994: 47)

Henrici/Riemer (1994) widmen der »Arbeit an phonetisch-intonatorischen Kenntnissen« beachtliche 15 Seiten. Die Suprasegmentalia (131 ff.) werden allerdings nur auf knapp drei Seiten (bei einem Umfang von 537 Seiten!) behandelt. Es werden die drei grundlegenden Intonationsmuster, die Wortbetonung bei einfachen und zusammengesetzten Wörtern sowie die Regeln für Hauptbetonung im Satz »in ruhiger Rede « beschrieben. Nicht behandelt werden u. a. Rhythmus, Pausen und emotionales Sprechen. Huneke/Steinig $\left(1997,{ }^{3} 2002\right)$ behandeln zunächst in einem sehr kurzen Kapitel »Phonetik und Phonologie" mit einer knappen Seite zu den Suprasegmentalia, zu denen sie Wortakzent, Satzintonation 
und Sprachrhythmus rechnen. Im Kapitel »Sprachliche Fähigkeiten « wird dann aber sehr detailliert auf zehn Seiten $»$ die Aussprache« (worunter auch die Suprasegmentalia gefasst werden) mit vielen praktischen Vorschlägen für den Unterricht behandelt. Sie plädieren für ein intensives Hörtraining vor dem eigentlichen Sprachkurs. Entwickelt werden soll vor allem »ein Gefühl für die Melodie, das Klangmuster und die Intonation der fremden Sprache [...] (Huneke/Steinig ${ }^{3}$ 2002: 140).

Storch (1999) widmet der Phonetik zehn Seiten. Es werden weitgehend nur die Segmentalia behandelt. Das gilt auch für die vorgestellten Übungstypen, wenn auch Wort- und Satzakzent und Intonation hin und wieder vorkommen. Nichtsdestotrotz teilt er die Auffassung,

»wonach dem suprasegmentalen Bereich als dem eigentlich primären die zentrale Rolle bei der Ausspracheschulung zukommt« (Storch 1999: 109).

Fazit: Phonetik bzw. Ausspracheschulung wird in allen erwähnten Didaktiken behandelt, der Anteil der Prosodie bzw. der Suprasegmentalia daran ist relativ gering trotz des Wissens über deren übergeordnete Bedeutung für den Sprachunterricht.

\section{Prosodie in Lehrwerken DaF}

Wir haben ca. 20 neuere Lehrwerke aus den letzten 20 Jahren auf ihren »Prosodiegehalt« durchgesehen. Wir können feststellen, dass seit etwa 1990 die Phonetik in den Lehrwerken zunehmend Berücksichtigung findet, so dass es eine große Ausnahme ist, wenn heute ein Grundstufen-Lehrwerk diesen Bereich nicht behandelt. Oft ist aber die Phonetik auch in neueren Lehrwerken quantitativ (1/4 oder gar 1/5 Seite pro Lektion) und auch, was die Qualität von Darstellung und Übungsformen angeht, nicht zufriedenstellend. Darüber hinaus wird die
Prosodie relativ wenig und oft auch zu spät behandelt. Weiterhin beschränkt sich der Prosodieanteil bei vielen Lehrwerken auf Wort- und Satzakzent, manchmal auch noch auf "Satzintonation«, womit i. d. R. die Tonhöhenbewegung am Ende eines Satzes (wir sagen: Ausspruchs) gemeint ist. Die folgenden wichtigen prosodischen Mittel werden nur selten behandelt:

- Akzentgruppen

- Pausen

- Langsames - schnelles Sprechen

- Emotionales Sprechen

- Interjektionen

Vorgestellt und geübt werden diese Themen nur in den Lehrwerken

- Dimensionen

- Mittelpunkt

- Moment mal

- Optimal

- Schritte

- Stufen International.

Keines der Lehrwerke beherzigt die Forderung, die auch die o. e. Didaktiken aufstellen, nämlich die Prosodie gleich von Anfang an und intensiv zu behandeln. Auch bei der Abfolge und Gewichtung der prosodischen Mittel ist noch großer Handlungsbedarf. Zur Behandlung des übergeordneten Themas Gesprochene Sprache in Lernzielbestimmungen, Didaktiken, Grammatiken und Lehrwerken für den DaF-Bereich siehe Vorderwülbecke (2008: 275 ff.).

\section{Was ist Prosodie?}

Begriffliche Klärungen

Hier sollen ganz kurz neben Prosodie auch Phonetik, Phonologie und Intonation näher bestimmt bzw. voneinander abgegrenzt werden:

\section{Prosodie}

Die Prosodie behandelt alle Eigenschaften der Lautkette, die die einzelnen Laute, die Segmentalia, überlagern. Bei der 
Prosodie ist es nicht möglich, Segmente festzumachen. Deshalb spricht man auch von Suprasegmentalia. Hierin liegt die besondere Komplexität der Prosodie als Untersuchungsgegenstand.

\section{Phonetik und Phonologie}

In der Phonetik ist die Zielrichtung eher beschreibend. In der Phonologie setzt man sich eher zum Ziel, Phoneme funktional $\mathrm{zu}$ beschreiben. Prosodie kann phonetisch oder phonologisch beschrieben werden. Je nach Ansatz ergeben sich unterschiedliche Terminologien. Die folgenden Ausführungen versuchen, eine Verbindung zwischen beiden Ansätzen herzustellen. Im DaF-Bereich gilt der Terminus Phonetik zumeist als Oberbegriff für Segmentalia und Suprasegmentalia.

\section{Prosodie - Intonation}

In der Literatur wird der Terminus Prosodie häufig als Synonym für Intonation verwendet. Unter Intonation versteht man allerdings im engeren Sinne nur die Tonhöhenbewegung (s. u.).

\section{Prosodische Eigenschaften}

Die drei wichtigsten prosodischen Eigenschaften sind:

- Dauer

- Tonhöhenbewegung

- Lautstärke.

Weitere Eigenschaften sind: Sprechgeschwindigkeit, Stimmqualität und Stimmdruck, Pausen und Rhythmus. Im vorliegenden Aufsatz beschäftigen wir uns hauptsächlich mit den oben genannten drei wichtigsten Eigenschaften.
Funktionen der Prosodie im Deutschen

Die prosodischen Eigenschaften werden im Deutschen auf verschiedenen Ebenen des Sprachsystems eingesetzt. Die wichtigsten sind:

- Wort

- Ausspruch

- Gespräch.

Wir legen im Folgenden den Schwerpunkt auf die Beschreibung der Funktionen der Prosodie auf Ausspruchsebene.

Prosodie im Wort (Wortakzent)

In jedem mehrsilbigen Wort wird eine Silbe stärker betont als die übrigen. Diese Silbe trägt den Wortakzent. Deutsch wird als Sprache mit freiem Wortakzent klassifiziert. Dies bedeutet, dass der Wortakzent auf unterschiedliche Silben fallen kann, z. B. ${ }^{1}$ :

\section{AUFenthalt \\ verLETZen \\ bäckeREI}

In Komposita fällt der Wortakzent in der Regel auf das Bestimmungswort, z. B.: GELDbeutel, KInokasse, unterSUchunsgegenstand. Es gibt auch Sprachen, in denen der Wortakzent normalerweise auf die gleiche Silbe fällt. Im Polnischen etwa fällt der Wortakzent normalerweise auf die vorletzte Silbe und im Französischen auf die letzte. Für Lerner mit diesen Ausgangssprachen, aber auch für Tonsprachensprecher ist der Wortakzent ein großes Lernproblem.

Je nach Sprache werden unterschiedliche prosodische Ressourcen eingesetzt, um den Wortakzent zu realisieren. Im Deutschen sind Lautstärke, höhere Artikulati-

1 Die Beispiele werden nach den folgenden Notationskonventionen angeführt. Wie in der Linguistik üblich, werden die betonten Silben durch Großschreibung gekennzeichnet. Der Rest wird klein geschrieben. Wenn in einem Satz mehr als ein Akzent vorliegt (z. B. ein Neben- und ein Hauptakzent), wird die Silbe, die den Hauptakzent trägt, durch Fettdruck angezeigt. Tonhöhenbewegungen werden entsprechend ihrer Richtung mit Schrägstrichen versehen. Die Tonhöhenbewegung in der letzten Silbe, d. h. im Grenzton, wird durch Pfeile wiedergegeben: $\searrow, \pi, \rightarrow$. 
onsspannung und Variation der Tonhöhe die Hauptkodierungsmittel des Akzents. In anderen Sprachen können bei der Akzentuierung andere prosodische Eigenschaften wichtiger sein. So spielen Lautstärke und Tonhöhenbewegung im Italienischen eher eine untergeordnete Rolle, während die Dauer der Sprachlaute die wichtigste prosodische Eigenschaft für die Kodierung des Akzents ist (vgl. Bertinetto 1981: 79-80, Missaglia 1997: 156). Im Deutschen kann die Lage des Wortakzents den Unterschied zwischen zwei Wörtern ausmachen: überSETZen vs. ÜBERsetzen.

Beim Aussprechen eines Wortes können Tonhöhenbewegungen realisiert werden. Diese werden als Übergänge von einem hohen Ton $\mathrm{zu}$ einem tiefen Ton oder umgekehrt oder als fallend-steigend realisiert z. B. /LEsen vs. LE\sen vs. /LE\sen. Tonhöhenbewegungen unterscheiden im Deutschen nicht zwischen zwei Lexemen. In den sogenannten Tonsprachen (z.B. im Chinesischen) wird hingegen diese prosodische Ressource lexemunterscheidend verwendet. Ein vergleichbares Phänomen findet sich im Deutschen nur bei den Interjektionen (siehe Ziff. 5.2). ${ }^{1}$

$\mathrm{Zu}$ den genannten Themen gibt es in Lehrwerken schon Übungen. Unter prosodischen Gesichtspunkten sollten folgende Themen vorrangig behandelt werden:

- Identifizierung des Akzents in mehrsilbigen Wörtern

- Identifizierung von Tonhöhenbewegungen in Wörtern (/LEsen vs. LE\sen)

- Bedeutungsänderung durch Verlagerung des Akzents

- Bedeutungsänderung bei Kompositabildung
Prosodie im Ausspruch

Unter Ausspruch wird das verstanden, was in der Grammatik der deutschen Sprache als »kommunikative Minimaleinheit« bezeichnet wird:

»Kommunikative Minimaleinheiten sind die kleinsten sprachlichen Einheiten, mit denen sprachliche Handlungen vollzogen werden können.« (Zifonun/Hoffmann/ Strecker 1997: 91)

Es kann sich um ein einzelnes Wort wie Achtung, um eine Wortkombination wie Keine Ahnung oder ich nicht oder um einen Satz handeln. Die wesentlichen prosodischen Eigenschaften des Ausspruchs sind:

- Haupt- und Nebenakzente

- Grenztöne.

In einem längeren Ausspruch werden unter allen Silben, die einen Wortakzent tragen, einige besonders stark betont. Haupt- und Nebenakzent(e) sind für die Gewichtung einzelner Informationsteile und auch für die rhythmische Gestaltung des Ausspruchs verantwortlich. Während die Wahl der Silbe, die den Wortakzent trägt, durch Konvention festgelegt ist (und im Wörterbuch nachschlagbar ist), hängt die Wahl der Silbe, die einen Neben- oder Hauptakzent trägt, von der Kommunikationssituation und von den Mitteilungsabsichten des Sprechers und seinen Hypothesen über den Wissensstand seines Adressaten ab. Ein Beispiel:

(1) (Was gibt's Neues von Maria?)

sie ist mit ihrem FREUND in SPAnien gewesen

Wenn der Sprecher davon ausgeht, dass der Adressat wissen möchte, ob es von Maria etwas Neues gibt, setzt er den Nebenakzent auf Freund und den Hauptakzent (durch Fettdruck hervorgehoben) auf das Wort Spanien. Der Hauptakzent folgt i. d. R. dem Nebenakzent bzw. den

1 Die eingerahmten Passagen enthalten Anregungen für den Unterricht. 
Nebenakzenten. Wenn es um die Person geht, mit der Maria in Spanien war, ist folgende Akzentzuweisung plausibel:

(2) (Mit wem ist Maria in Spanien gewesen?)

(sie ist) mit ihrem FREUND (in spanien gewesen)

Die Akzente dienen also dazu, die für die Kommunikation wichtigsten Informationsabschnitte hervorzuheben. Die Beziehungen zwischen Akzentsetzung und Information werden in Abschnitt 3. »Informationsstruktur « behandelt.

Auf Ausspruchsebene nehmen die sogenannten Grenztöne wichtige Funktionen wahr. Dies sind Tonhöhenbewegungen am Ende eines Ausspruchs (siehe Ziff. 4.3). Die Richtung des Grenztons zusammen mit Lage und Art des Hauptakzents spielen für die Kodierung der Satzarten im Deutschen eine wichtige Rolle (vgl. Graffmann/Moroni 2007).

Die Zuordnung bestimmter intonatorischer Muster zu den unterschiedlichen Satzarten hat Tradition im Phonetikunterricht. Danach steht die terminale oder auch fallende Intonation für den einfachen Aussagesatz, den Imperativsatz, den Wunschsatz und die W-Frage. Grenztöne zeigen wir mit einem fallenden bzw. steigenden Pfeil am Ende des Ausspruchs an:

(3) das ist MEI $\backslash$ ne entscheidung $\searrow$ (Aussagesatz)

(4) GLAU $\backslash$ be daran $\$ (Imperativsatz)

(5) hätte ich dir doch verTRAUT $\backslash \searrow$ (Wunschsatz)

(6) wie HIE $\backslash$ ßen diese männerУ (WFrage)

Dieses Muster kann als Kombination von einem fallenden Hauptakzent und einem fallenden Grenzton beschrieben werden. Die steigende Intonation steht für die Entscheidungsfrage und die Nachfrage:

(7) /KOMMST du heute abend mit $\pi$ (Entscheidungsfrage)
(8) heute abend kommt /WER mit (Nachfrage)

Oft wird diese Kontur (steigender Hauptakzent und steigender Grenzton) auch »interrogativ« genannt. Diesen Begriff sollte man im Sprachunterricht vermeiden, weil die Lerner dann fälschlicherweise annehmen, dass alle Fragefunktionen mit steigender Intonation realisiert werden bzw. dass steigende Intonation immer Fragefunktion signalisiert.

Die progrediente oder auch schwebende Intonation kennzeichnet Unabgeschlossenheit z. B. in Satzgefügen und bei Aufzählungen:

(9) wenn du /MITkommen kannst $\rightarrow$ komme ich $\mathrm{AUCH} \backslash$ mit $\searrow$

Die Lehrenden sollten im Unterricht klar machen, dass es sich hier um Grundmuster handelt, die im konkreten Fall auf vielfältige Weise abgewandelt werden können. Oppenrieder (1988: 186), aber auch andere Autoren sprechen von »intonatorischen Prototypen«.

Stock (1996: $96 \mathrm{ff}$.) und andere Autoren zeigen auf, dass insbesondere beim freien Sprechen Faktoren (z. B. die Situation mit dem besonders wichtigen Sprecher-Hörer-Bezug) ins Spiel kommen, die das obige System durchbrechen.

Die fallende Intonation der W-Frage wird hauptsächlich beim Vorlesen von sachlichen Texten eingesetzt, wohingegen die steigende Intonation vor allem in spontanen Gesprächen vorkommt. Die steigende Intonation bei der W-Frage in Beispiel (10b) nennen Stock (1996) und andere Autoren »Kontaktintonem «.

(10a) warum KOMMST \du nicht $\$ (prototypisch sachlich/neutral)

(10b) warum KOMMST/ du nicht $\pi$ (mit besonderer Zuwendung, freundlich)

Die Entscheidungsfrage kann einmal mit einem steigenden und einmal mit einem 
fallenden Hauptakzent realisiert werden. In beiden Fällen folgt ein steigender Grenzton am Ende des Ausspruchs:

(11a) Typ 1: haben sie die /ZEUgen vernommen $\pi$

(11b) Typ 2: haben sie die ZEU \gen vernommen $\pi$

Typ 1 und Typ 2 sind im Diskurs nicht auswechselbar. In Typ 1 wird eine einfache Bestätigung erwartet, in den meisten Fällen durch $j a$ oder nein. In Typ 2 rechnet der Sprecher damit, dass der Adressat sich nicht auf das einfache »ja « beschränkt, sondern dass er eine zusätzliche Erklärung oder Frage anschließt, z. B. ob die Vernehmung erfolgreich war.

Danach hängt die Intonation der Entscheidungsfrage auch vom Status der Frage innerhalb einer Frageaktivität ab. Die Prosodie wird in diesem Fall nicht durch die Satzart bestimmt, sondern durch die Erwartungshaltung des Sprechers. Typ 1 kommt in der gesprochenen Rede dreimal häufiger vor als Typ 2 (Selting 1995: 232 ff.; Schwitalla 2006: 68).

Im Unterricht sollten folgende Themen behandelt und geübt werden:

- Lage des Hauptakzents. Die Lehrperson gibt einen kleinen geschriebenen Text vor, in dem keine Akzente eingetragen sind. Anhand einer Tonaufnahme dieses Textes sollen die Lerner die Hauptakzentsilbe markieren, z. B. doppelt unterstreichen.

- Opposition zwischen Haupt- bzw. Nebenakzent. Die Lehrperson gibt den Lernern das Transkript eines Ausschnitts aus einem authentischen Gespräch und spielt die Tondatei vor. Die Lerner sollen nach mehrmaligem Anhören Haupt- und Nebenakzente ermitteln und diese markieren (z. B. durch einfache oder doppelte Unterstreichung oder durch unterschiedliche Farben).
- Unterschied bei W-Fragen zwischen sachlich/neutral und höflich/freundlich. Die Lerner hören mehrere W-Fragen und entscheiden, ob die jeweilige Frage sachlich/neutral oder höflich/freundlich gestellt ist, indem sie im Transkript nach der letzten Silbe einen Pfeil nach oben bzw. unten einzeichnen.

- Status von Entscheidungsfragen. Übungen zum Unterschied von Typ 1 und Typ 2 müssen in einem Fragesatz die Redeabsicht des Sprechers zum Ausgangspunkt machen: Sprecher erwartet kurze Antwort vs. Sprecher erwartet ausführliche Antwort. Die Lehrperson kann z.B. Fragen von Typ 1 oder Typ 2 (auch von einem Tonträger) vorgeben, die Lerner müssen dann die Erwartungshaltung des Sprechers »erhören« und die jeweils passende kurze oder ausführliche Antwort geben.

Prosodie im Gespräch

Seit Mitte der 1980er Jahre hat sich im Zuge der Studien zur Konversationsanalyse eine Prosodieforschung entwickelt, bei der die Rolle prosodischer Eigenschaften für die Gesprächsorganisation in den Vordergrund gerückt wird. Hierbei werden u. a. prosodische Signale untersucht, die dazu beitragen, den Redewechsel zu steuern. So kann eine steigende oder fallende Tonhöhenbewegung das Ende eines Gesprächsbeitrags signalisieren und dem Gesprächspartner die Gelegenheit geben, das Wort zu ergreifen. Eine leicht steigende bzw. progrediente Tonhöhenbewegung indiziert hingegen, dass der Sprecher das Rederecht beibehalten möchte. Einen weiteren Schwerpunkt der konversationsanalytisch orientierten Prosodieforschung bildet die Rolle prosodischer Eigenschaften bei der Interpretation von konversationellen Aktivitäten. So kann der gleiche 
Gesprächsbeitrag je nach »prosodischer Verpackung « (Auer/Selting 2001: 1125) ganz unterschiedliche Reaktionen seitens des Gesprächspartners auslösen. Günthner (1996) zeigt, dass wieso-/warum-/weshalb-Fragen, wenn prosodisch markiert (z. B. durch erhöhte Lautstärke und steigend-fallende Tonhöhenbewegung), als Vorwurf interpretiert werden.

In all diesen Fällen geht es um Funktionen in der konkreten Interaktion oder um kommunikative Bedeutungen. Die sprachlich-prosodischen Mittel dafür müssen erlernt werden. Die Bedeutung der Prosodie für die Gesprächsorganisation und die Kenntnis der unterschiedlichen prosodischen Mittel harrt allerdings weiterhin der Umsetzung in die Praxis des DaF-Unterrichts.

Wünschenswert sind Übungen, in denen der Lerner prosodische Signale als gesprächsdeterminierend interpretiert, z. B. »der Sprecher will weiterreden « vs. »der Sprecher hat alles gesagt, was er sagen wollte«. Dies könnte man z. B. mit Dialogen auf Tonträger üben, indem man an den »Nahtstellen « stoppt und die Lerner entscheiden lässt, ob der Redebeitrag zu Ende ist oder weitergeht. Es wäre gut, wenn Übungsformen entwickelt würden, mit denen man die kommunikative Bedeutung prosodisch unterschiedlich gestalteter Redebeiträge erarbeiten könnte.

Im Rahmen der Gesprächsforschung wurde das Gesprächsanalytische Transkriptionssystem (Selting/Auer/Barden/ Bergmann/Couper-Kuhlen/Günthner/ Quasthoff/Meier/Schlobinski/Uhmann 1998) entwickelt. Es handelt sich um Konventionen zur Transkription authentischer gesprochener Sprache. Im Fortgeschrittenen-Unterricht könnte man

(i) diese Konventionen einüben, z. B. für Pausen, Haupt-, Nebenakzente und Grenztöne, (ii) Gespräche und die dazugehörigen Transkripte anhören bzw. auf einzelne prosodische Eigenschaften hin analysieren. Dies fördert das prosodische Sprachbewusstsein.

\section{Informationsstruktur}

Situation und Informationsstruktur

Die Informationsstruktur stellt die Aufbereitung einer Information in einer kommunikativen Situation dar. Situationen lassen sich definieren durch

- den Inhalt der Kommunikation

- die Intention des Sprechers

- den Sprecher-Hörer-Bezug

- die Wichtigkeit

- die sprachliche Ebene, auf der die Kommunikation stattfindet.

(Weiteres zur Bedeutung der Kommunikationssituation in 5.1).

Die sprachlichen Mittel, Syntax, Prosodie etc., die der jeweilige Sprecher für seine Aussprüche einsetzt, variieren je nach der Situation, in der die Kommunikation stattfindet, und sie dienen dem »Zweck der Optimierung des Informationstransfers im Diskurs« (Féry 2009). Folgendes Beispiel zeigt, wie die Lage des prosodischen Mittels Akzent eine Aussage verändern kann:

(12) (Außenminister Steinmeier sagt zu den Vorwürfen an ihn im Fall Kurnaz,)

er habe nichts geTAN

(Die Opposition wirft ihm aber vor,) er habe NICHTS getan

Brückenkontur als Grundmuster

In dem folgenden Beispiel:

(13) mein /AUto hat eine PAN $\backslash$ neע

ist Auto das Wort, über das etwas ausgesagt wird. Die Tonhöhenbewegung nach oben kennzeichnet Auto als Topik. Diese Tonhöhenbewegung ist also ein TopikMarker. Syntaktisch ist das Topik zu er- 
kennen an der Linksstellung im Ausspruch.

Panne ist das Wort, das den Kern der Information kennzeichnet. Die Tonhöhenbewegung nach unten kennzeichnet Panne als Fokus. Diese Tonhöhenbewegung ist also ein Fokus-Marker. Syntaktisch ist der Fokus zu erkennen an der Rechtsstellung im Ausspruch. Auf diese Weise wird die Gewichtung innerhalb des Ausspruchs geregelt und damit die konkrete Informationsstruktur.

Die Tonhöhenbewegung der Äußerung Mein /AUto hat eine PAN \ne \lässt sich in Form einer Brücke darstellen: ${ }^{--} \backslash$. Man spricht deshalb von einer »Brückenkontur « (Féry 1993: 149). Diese lässt den Ausspruch als abgeschlossen erscheinen. Die Brückenkontur stellt ein Grundmuster für Tonverläufe in Aussprüchen dar. Andere Konturen lassen sich in ihrer Abweichung von diesem Muster beschreiben. Deshalb ist es nützlich, das Muster Brückenkontur von Anfang an im Bewusstsein der Sprachlerner zu verankern.

In Übungen werden anhand eines Beispielsatzes (mein /AUto hat eine PAN $\backslash$ ne $У$ ) andere Sätze gesprochen, in denen die Lerner die Tonhöhenbewegungen der Brückenkontur deutlich nachvollziehen.

Hauptakzent und besondere Fokussierung

Topik und Fokus tragen Akzente, wobei der Fokusakzent der stärkere ist. Für diesen Akzent hält die Didaktik unterschiedliche Begriffe bereit wie Satzakzent, Äußerungsakzent, Satzkern, Hauptakzent, Hochakzent u. a. Wir verwenden »Hauptakzent«. Durch die starke Hervorhebung des Fokus wird der Adressat in die Lage versetzt, die wichtigste Information in der Äußerung klar zu identifizieren.
Übungen, die Fokus-Hervorhebung sowohl beim Lesen als auch beim freien Sprechen trainieren, sind von Anfang an unerlässlich. Umgekehrt soll der Sprachlerner als Hörer sich daran gewöhnen, diesem stärksten betonten Wort im Ausspruch seine besondere Aufmerksamkeit zuzuwenden. Es hat sich bewährt, dass man Sprachlerner auf jeder Stufe vor dem Lesen eines Textes dazu auffordert, in jedem Ausspruch die Silbe, die den Hauptakzent trägt, zu markieren. Das sinnerfassende Lesen wird dadurch stark gefördert.

Befindet sich der Fokus einer Äußerung und damit der Hauptakzent nicht am Ende, sondern weiter vorne im Ausspruch, so spricht man von besonderer Fokussierung. Ein Beispiel:

(14) ich möchte DEN apfel

In diesem Beispiel wird deutlich, dass Äpfel schon im Aufmerksamkeitsbereich des Sprechers vorhanden sind, dass es ihm aber darum geht, aus der Menge der Äpfel einen bestimmten auszuwählen. Die besondere Fokussierung wird in den meisten Fällen nicht durch die Syntax, sondern ausschließlich über die Prosodie realisiert. Der Sprecher drückt durch die Akzentuierung aus, worauf es ihm ankommt.

In Hörübungen zur besonderen Fokussierung werden die Lerner aufgefordert zu identifizieren, worauf es dem Sprecher ankommt. Dabei treffen sie eine Auswahl aus mehreren theoretisch möglichen Betonungen. In Sprechübungen sollen sie selbst durch deutliche Akzentsetzung ihre jeweilige Intention ausdrücken.

Zur Fokussierung können auch andere Elemente dienen, die dazu beitragen, die Informationsstruktur zu kodieren. So wurde beobachtet, dass Abtönungsparti- 
keln dazu tendieren, unmittelbar links vom Fokus zu stehen (vgl. Lerner 1987; Büring 2006; Moroni 2006). Auf diese Weise fungieren Modalpartikeln als eine Art informationsstrukturelle Anzeiger. Ein Beispiel:

(15) A: hans ist nicht DA \MERK \würdig

B: der hat doch UR \laub

Bei Vorlese-Übungen kann man Lerner darauf hinweisen, dass unmittelbar rechts von einer Abtönungspartikel ein fallender Akzent bzw. ein Fokus folgen muss. Dazu kann man z.B. die Abtönungspartikeln im Text vorher markieren lassen.

Der Fokusakzent kann auch auf dem Verb liegen:

(16) (ich sagte dir doch,) er WAR damals in köln

In diesem Fall möchte der Sprecher ausdrücken, dass er darauf beharrt, dass der Tatbestand zutrifft. Er will Recht haben. Dieser Akzent wird in der Fachliteratur (vgl. Höhle 1988) als »Verum-Fokus« bezeichnet. Das Beharren auf der eigenen Position hat einen wichtigen Stellenwert in der Kommunikation. Übungen zum Verum-Fokus (den man im Unterricht »Verbbetonung « nennen könnte) sind selten in Lehrwerken anzutreffen, sollten aber einen größeren Stellenwert erlangen.

Thema-Rhema und Topik - Fokus

Im Sprachunterricht wird die Gliederung von Sätzen - wenn überhaupt - im Wesentlichen über die sogenannte ThemaRhema-Gliederung vorgenommen, wobei Thema für den bekannten Teil der Information und Rhema für die neue Information steht. Diese Gliederung erleichtert textgrammatische Arbeitsweisen und hat sich in der Sprachdidaktik bewährt. Im Gefolge dieser Überlegungen trifft man häufig auf die Meinung, dass das Rhema den Hauptakzent im Satz trage. Es hat sich nun gezeigt, dass die Thema-Rhema-Gliederung nicht 1:1 auf die Prosodie übertragbar ist und auch das Thema einen Hauptakzent tragen kann. Hierzu zwei Beispiele aus Lötscher (1983: $134 \mathrm{ff}$.$) :$

(17) das BAD ist frei

(18) die POST ist gekommen

Im folgenden Beispiel

(19) (Die Familie erschien zum Essen.) nur KARL fehlte

ist im Sinne der Thema-Rhema-Gliederung Karl das Thema des zweiten Satzes. Nach der Regel der Rhema-Betonung dürfte es nicht den Hauptakzent tragen. Mit dieser Akzentsetzung hebt der Sprecher eine Person aus der Gruppe der Familienmitglieder besonders hervor. Deshalb trägt Karl einen Hauptakzent in Form eines fallenden Akzents.

Für den Tatbestand, dass aus einer Menge bekannter thematischer Elemente ein einzelnes Element ausgewählt wird, prägt Büring (2006: $155 \mathrm{ff}$.) den Begriff des »Kontrast-Topiks«. In Lehrwerken finden wir dafür den Ausdruck »Kontrastakzent «.

Übungen zum Kontrastakzent haben häufig das Schema »nicht der, sondern der«. Die Hervorhebung eines Elements aus einer Menge wie im obigen Beispiel kommt seltener vor, verdient aber mehr Berücksichtigung.

Der Kontext kann auch dafür verantwortlich sein, dass Wörter bzw. Begriffe betont werden, die nur im Bewusstsein bzw. Gedächtnis der Kommunikationspartner in einer gegebenen Situation vorhanden sind. Ein Beispiel:

(20) (Karin liegt mit Grippe im Bett. Martin besucht sie. Kaum ist Martin eingetreten, ruft ihm Karin entgegen:) das schlechte WETter ist schuld 
Im Kontext ist klar, dass es einen Grund für das Kranksein geben muss. Weiterhin wissen beide, dass in den letzten Tagen schlechtes Wetter war. Durch die Betonung auf »Wetter « nimmt Martin direkt Kontakt auf, indem er auf die Ursache der Erkrankung von Karin fokussiert.

Bei Übungen zu diesem Problem muss es immer darum gehen, einen Kontext zu definieren, in dem ein zeitlicher Abstand von einer Situation A zu einer späteren Situation B besteht, wobei der Sprecher in Situation B an die frühere Situation A anschließt.

Weiterhin beeinflussen emotionale Faktoren Tonhöhenverlauf und Akzentsetzung in den verschiedenen Satzarten, wie u. a. schon im Zusammenhang mit dem Kontaktintonem erläutert. Dazu siehe auch Abschnitt 5.

\section{Rhythmische Einheiten}

\subsection{Rhythmus und Rhythmisierung}

Für Rhythmus in der Sprache gibt es keine allgemein akzeptierte Definition. Das erschwert auch die Behandlung von Rhythmus in Lehrwerken und im Unterricht (vgl. Bose 1999: 225). Die Rhythmisierung eines Ausspruchs entsteht durch die Sprechmelodie, die Akzente in Wort und Satz, durch den starken Spannungsunterschied zwischen akzentuierten und akzentlosen Silben sowie durch die Untergliederung in kleinere rhythmische Einheiten. Jedes Wort ist schon eine rhythmische Einheit und kann ja auch einen vollständigen Ausspruch realisieren (wie z.B. das Wort Natürlich in Kommst du auch? - Natürlich). Wir konzentrieren uns hier aber auf die für die Konstitution von Rhythmus in Aussprüchen und für den Sprachunterricht relevanteren Einheiten Akzentgruppe (AG) und Intonationsphrase (IP).

\subsection{Akzentgruppen}

\section{Terminus und Definition}

Für »Akzentgruppe « gibt es verschiedene andere Bezeichnungen: Die Grundzüge einer deutschen Grammatik (Heidolph/Flämig/Motsch 1981: 852) und die Grammatik der Deutschen Sprache (Zifonun/Hoffmann/Strecker 1997: 215) nennen sie »Takt«. Stock/Hirschfeld (1996: 33) und das Lehrwerk Dimensionen bezeichnen sie als »rhythmische Muster bzw. »Rhythmusmuster«. Im Lehrwerk Stufen International heißen sie »Rhythmusgruppen «.

Eine AG hat immer mindestens eine Akzentsilbe und fakultativ eine oder mehrere unakzentuierte Silben, die der Akzentsilbe vorangehen oder folgen können. Der Bezugsbereich bzw. die »Akzentdomäne « (Zifonun/Hoffmann/Strecker 1997: 218) ist also relativ eng begrenzt. Die AG bildet eine feste melodisch-rhythmische Einheit, die nicht durch Sprechpausen auseinander gerissen werden darf. Eine minimale AG besteht nur aus einem Wort bzw. einer Silbe (wie das Oh in Oh, da kommt er ja.) Eine maximale AG umfasst eine Intonationsphrase oder einen ganzen Ausspruch (Da laufen sie!).

Die Anzahl der im konkreten Fall realisierten Akzente und damit AGn hängt vom Sprechstil ab. In normaler, ruhiger Sprechweise können mehr Akzente gesetzt werden als in schneller, oft emotionaler Sprechweise, in der z. B. zwei AGn in eine IP eingehen.

(21a) | du musst dich | beim nächsten mal | viel massiver | reinhängen |

(21b) I du musst dich beim nächsten mal | viel massiver reinhängen |

Die Grenze einer AG kann durch eine Pause oder durch den Zusammenfall mit einer syntaktischen Einheit signalisiert werden (z. B. einer Nominalphrase). Und natürlich spielt auch die Intention des Sprechers eine Rolle. 


\section{Isochronie}

Isochronie bezeichnet die Tatsache, dass die Sprechdauer von einer Akzentsilbe zur nächsten potenziell gleich lang ist, und zwar unabhängig von der Zahl der unbetonten Silben dazwischen. Diese Isochronie ist von deutschen Muttersprachlern als festes (Erwartungs- und Produktions-)Muster gespeichert, auch wenn die Abfolge der Akzentsilben oft nicht in vollkommen zeitgleichen Abständen erfolgt. Dieses isochronische Muster verleiht dem Deutschen eine relativ hohe rhythmische Gleichmäßigkeit. Man spricht deshalb von einem StaccatoRhythmus des Deutschen.

Die Realisierung dieses Zeitgleichheitsmusters wird dadurch erreicht, dass im Deutschen sehr viel artikulatorische Spannung auf die Akzentsilbe gelegt wird und entsprechend sehr wenig Spannung auf die unakzentuierten Silben. Die Akzentsilbe wird lauter, relativ länger und damit deutlicher - oft auch höher - gesprochen als die unbetonten Silben, die leiser, schneller und artikulatorisch weniger präzise gesprochen werden. Dadurch ergeben sich bei letzteren viele Lautangleichungen, Reduktionen und Elisionen.

\section{Die AGn im Unterricht}

Das Deutsche gehört bekanntlich zur Gruppe der akzentzählenden Sprachen wie z.B. auch das Englische. $\mathrm{Zu}$ den silbenzählenden Sprachen gehören die romanischen und viele asiatische Sprachen. Lerner aus diesen Sprachgruppen haben große Probleme, die rhythmische Struktur des Deutschen und besonders auch AGn zu erkennen und vor allem zu produzieren. Deshalb muss der Lernbereich Prosodie mit den rhythmischen Eigenschaften im Zentrum von Anfang an und intensiv erarbeitet werden. Die AG muss als rhythmischer Grundbaustein von Aussprüchen verinnerlicht werden.
Diese Prioritätensetzung müsste auch eine »neuartige Strukturierung des Sprachmaterials« zur Folge haben (vgl. Veličkova 1999: 223).

Durch die Arbeit am Rhythmus schon im Anfängerstadium können sich die Lernenden darüber hinaus von der Fixierung auf Buchstaben bzw. Einzellauten und Wörtern als Basiseinheiten der Perzeption und Produktion lösen und zu den prosodisch viel wichtigeren AGn gelangen.

Ganz zu Anfang müsste - quasi als Vorübung - der große Unterschied zwischen der Akzentsilbe und den sie umgebenden unbetonten Silben intensiv »erhört« und erlernt werden zum Beispiel durch Klopfen und Klatschen. Hilfreich sind aber auch Bewegung im Raum, z.B. Gehen mit kurzem Aufstampfen bei Akzentsilben sowie die Verwendung von Rhythmusinstrumenten wie Triangel, Reibeholz und Trommel. Erst danach sollte man AGn in Verbindung mit sprachlichen Einheiten vorstellen und üben, z. B. mit kurzen Sprechstücken, Rhythmen oder Raps (dazu und zu den Rhythmusinstrumenten vgl. Fischer 2007).

Durch Demonstration und Übung sollte man auch klar machen, dass (mehrsilbige) Wörter rhythmisch die gleiche Struktur wie AGn haben, und dass beide fest gefügte melodisch-rhythmische Einheiten sind. Denn bei Wörtern käme niemand auf die Idee, sie durch eine Sprechpause zu trennen:

$\begin{array}{ccc}\text { o } \bullet & \text { o } \text { o } \bullet \quad \text { o } 0 \\ \mid \text { pass AUF! | | das war VORgestern | } \\ \text { muSIK | | oberAMmergau | }\end{array}$

Sehr gut eignet sich auch die Arbeit mit zweiteiligen Redewendungen oder Sprichwörtern wie
a) Morgenstund' hat Gold im Mund.
b) Heute so, morgen so.
c) Wie du mir, so ich dir. 
Am Beispiel b) kann man dazu zeigen, dass durch Variation der rhythmischen Gestalt verschiedene Bedeutungen entstehen (heute SO, morgen SO; HEUte so, MORgen so.)

Vor allem, wenn ein Text vorgelesen oder mündlich nacherzählt werden soll, ist es hilfreicher, anstelle von Stichwörtern AGn als Gerüst anzubieten z.B. durch eine unterstützende grafische Gestaltung:

| eines TAges | LIEF | eine durstige ZIEge | zum BRUNnen. |

Durch eine andere grafische Anordnung kann man noch deutlicher machen, dass jede AG ungefähr die gleiche Sprechdauer beansprucht:

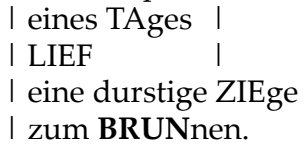

\subsection{Intonationsphrasen}

Die größeren rhythmischen Teileinheiten, in die Aussprüche durch prosodische Mittel gegliedert werden können, werden üblicherweise Intonationsphrasen (IPn) genannt. Diese können aus einer oder mehreren Akzentgruppen bestehen.

Über die Eigenschaften, die IPn kennzeichnen, besteht in der Forschung keine Einigkeit. Einig ist man sich lediglich darüber, dass in einer IP ein prominenter Akzent vorliegen muss. Diese Akzentsilbe hat einen weiteren Bezugsbereich als die Akzentsilbe einer AG, d. h. der IPAkzent kann in seinem Bezugsbereich eine (schwächere) Akzentsilbe haben. Hier ein Beispiel:

(22) I I der mieter nebenAN hat sich SCHON entschuldigt || aber mit was für einem geSICHT | |

Es liegen zwei IPn vor: der mieter nebenan hat sich schon entschuldigt und aber mit was für einem Gesicht. Die erste IP basiert auf zwei AGn: der mieter nebenan (mit einem potenziellen »Nebenakzent « auf $A N$ ) und hat sich schon entschuldigt. Auf der Ausspruchsebene wird dann im konkreten Fall festgelegt, welcher der beiden IP-Akzente den Hauptakzent des Ausspruchs ausmacht. I. d. R. ist das der jeweils letzte. Es reicht allerdings nicht, nach den IPAkzenten zu suchen, um die Gliederung von Aussprüchen in IPn festzuhalten. Notwendig sind auch Kriterien, nach denen die Grenzen der IPn ermittelt werden. Die wichtigsten Kriterien sind die folgenden (vgl. Féry 1993: 59-60):

Die Grenze einer IP fällt meist mit einer syntaktischen Grenze zusammen, z. B. Satz- oder Teilsatzgrenze. Grenzsignal kann auch eine Pause sein (vgl. Uhmann 1991: 121). Die rechte Grenze einer IP ist in der Regel durch eine deutliche (meist steigende) Tonhöhenbewegung gekennzeichnet. Je mehr Kriterien zutreffen, desto eindeutiger lassen sich die Grenzen bestimmen.

Die Aufteilung in IPn kann darüber hinaus mehrdeutige syntaktische Strukturen eindeutig machen wie im folgenden Beispielpaar (vgl. Peters 2005):

(23a) I I Ich bedaure heute nicht I I in Frankreich studiert zu haben I|

(23b) | | Ich bedaure heute || nicht in Frankreich studiert zu haben I |

Um die Verwechslung mit der syntaktischen Phrase zu vermeiden, könnte man im Unterricht z. B. »Intonationsgruppe« verwenden. Für das Bewusstmachen der IP im Unterricht bietet sich an, Texte nach dieser rhythmischen Einheit grafisch anzuordnen:

| | Einem reichen Manne wurde seine Frau krank, I I

I I und als sie fühlte, dass ihr Ende herankam, I I

I I rief sie ihre einzige Tochter zu sich ... I 
Dieses Vorgehen macht hier besonderen Sinn, weil die IP-Grenze ja oft durch eine Pause gekennzeichnet ist, die dann auch eine Atempause darstellt. Damit wird ein flüssiges Vorlesen oder (Nach-)Erzählen gefördert. Und zusätzlich wird in vielen Fällen die syntaktische Struktur visuell verdeutlicht.

\section{Prosodie und Emotion}

\subsection{Prosodische Ausdrucksmittel der Emotion}

Ausdruck von Emotion

Das Verhältnis von Sprechern und Hörern ist immer von affektiven Reaktionen begleitet. Je nachdem in welcher Beziehung sie zueinander stehen, variieren sie die prosodische Gestalt. Die traditionelle Fremdsprachendidaktik ignoriert diese Tatsache weitgehend. Ihr liegt das »Bild des geplant handelnden und kognitiv bestimmten Menschen « (Fiehler 1990: 23) zu Grunde, wobei der schriftliche Ausdruck, zugeschnitten auf den einfachen Aussagesatz, die hauptsächlichen Parameter liefert. In der alltäglichen Kommunikation ist die sachlich-neutrale Sprechweise keineswegs die Norm. Es kann deshalb nur um eine Unterscheidung zwischen Sprechweisen mit schwach oder mit stark ausgeprägten Emotionen gehen.

Emotionales Sprechen ist normalerweise mit einer Steigerung der Spannung des Artikulationsapparates verbunden, die sich z. B. in einer Veränderung der Akzentverteilung im Ausspruch manifestiert. Solche Spannungserscheinungen werden vom Kommunikationspartner als Ausdruck von Emotionalität wahrgenommen. Darüber hinaus wirken sich Emotionen auf den ganzen Körper aus. Sie haben Einfluss auf Körperhaltung, Gesichtsausdruck, Gestik-Mimik und können körperliche Reaktionen wie Zittern oder Erröten auslösen. (Über die korporalen Steuerungsprozesse vgl. Scherer/Wallbott 1990).

Die prosodischen Mittel zum Ausdruck der Emotion sind Akzente, Tonhöhenbewegung, Sprechgeschwindigkeit, Rhythmus, Pausen, Stimmlage sowie Klang der Stimme. So zeichnet sich z.B. Trauer durch tiefe Stimmlage, langsame Sprechgeschwindigkeit und Dehnung einzelner Silben aus.

Bei der Beschreibung sogenannter Basisemotionen beziehen sich die meisten $\mathrm{Au}-$ toren auf die Kategorien Tonhöhenbewegung, Akzente, Tempo (Tischer 1993: 110 ff., Kehrein 2002: 137). Mit Hilfe dieser drei Kategorien lassen sich emotionale Ausdrucksweisen ebenfalls im Sprachunterricht beschreiben und üben.

\section{Emotion im Unterricht}

Schwerdtfeger (1997: 587) plädiert dafür, »Emotionen als integrale Bestandteile des Fremdsprachenunterrichts $\mathrm{zu}$ verankern « und damit den Fremdsprachenunterricht »in neue Bahnen « zu lenken. Lernende sollen die Bereitschaft entwickeln, ihre Gefühle auch im Fremdsprachenunterricht auszudrücken. U. E. ist diese über zehn Jahre alte Forderung kaum in die Realität umgesetzt worden.

Reinke (2007: 18-32) hat auf der Basis von vorhandenen Übungen einen Katalog mit acht Forderungen entwickelt, an denen sich Lehrer und Autoren orientieren können. In diesen Forderungen wird ähnlich wie bei Schwerdtfeger vor allem das Prinzip deutlich, dass das Lernen des emotionalen Sprechens in den Lernzielkatalog des Fremdsprachenunterrichts gehört, und dass das Bewusstmachen gewisser Regularitäten für emotionales Sprechen von Anfang an ein Unterrichtsinhalt sein müsste. Dabei sollte soweit wie möglich das Lernen emotionaler Ausdrucksweisen verknüpft werden mit anderen Kommunikationsmitteln wie Mimik und Gestik oder stotterndem 
Sprechen. Dazu gehören auch grammatische und lexikalische Ausprägungen emotionaler Intentionen wie z. B. die Verwendung von Interjektionen (siehe Ziff. 5.2). Dabei geht es weniger darum, diese selbst zu produzieren, sondern vielmehr darum, sie als Ausdrucksmittel zu entdecken und zu unterscheiden. Reinke erhebt die Forderung, dass den Lernenden Raum gegeben werden muss, »über ihre Wahrnehmungen [...] zu reflektieren « (Reinke 2007: 17 f.). Die Sensibilisierung für Artikulationsweisen wie Flüstern, Klangfülle, helle oder dunkle Klangfarbe, Expressivität, behauchte Stimme (hierzu Scherer 1982) trägt dazu bei, dass die Lernenden den Klang der deutschen Sprache immer deutlicher erfahren. Sprecherziehung und Theaterpädagogik liefern hier wichtige Anregungen. U. E. bewirkt emotionales Sprechen eine Dynamisierung des Unterrichts und trägt dazu bei, die Sprechsituation authentisch zu machen.

Für die Prosodie emotionaler Ausdrucksweisen gibt es keine bewährten Übungstypologien. Ausgangspunkt für eine Lehre des emotionalen Sprechens sollte nicht der einzelne sprachliche Ausdruck sein, sondern vielmehr die kommunikative Situation, denn: »Emotionen [...] sind sozial geregelt« (Fiehler 1990: 2). In der Situation wird deutlich, warum der einzelne Sprecher ängstlich, freudig o. Ä. ist. Hieraus ergibt sich die Forderung, authentische Kommunikationssituationen zwischen den Lernenden in den Unterricht $\mathrm{zu}$ integrieren und die Regeln für emotionales Sprechen zu »vermitteln, diskutieren und trainieren « (Reinke 2007: 21). Dabei kann ein Lehrwerk nur begrenzt Hilfestellung leisten, z. B. mit textbegleitenden Fotos oder Zeichnungen oder auch mit Angabe von Emotionen vor dem jeweiligen Redebeitrag wie in Drehbüchern. Sehr geeignet sind Video-Sequenzen aus Spielfilmen oder Fernsehsprachkursen (z. B. »Einblicke«). Textsorten, in denen Emotionen gezielt eingesetzt werden, wie z. B. Werbung oder politische Reden, sollten insbesondere im Hinblick auf den emotionalen Ausdruck geübt werden und in ihren Wirkungen diskutiert werden.

\subsection{Interjektionen}

\section{Überblick}

Die Interjektionen sind syntaktisch autonom und haben damit Eigenschaften von Sätzen. Sie können Sprechakte realisieren und sind meist "expressiver Ausdruck einer spontanen Emotion « (Nübling 2004: 13). Den ganzen Umfang ihrer kommunikativen Funktionen fasst Schwitalla so zusammen:

»Sie [die Interjektionen] betreffen Konsens und Dissens, die Herstellung des Kontakts und die Regelung der Beziehung, den Ausdruck von Gefühlen und (wie Modalpartikeln) die Einstellung zum Inhalt des Gesagten und den Vergleich der gewonnenen Mitteilungen mit eigenem Wissen.«(Schwitalla 1997: 175)

Die Liste der Interjektionen und deren Lexikalisierungsgrad ist offen, weil immer wieder neue gebildet werden und andere außer Gebrauch geraten. Nübling (2004: 15) listet die folgenden prototypischen primären Interjektionen auf (Die mit * versehenen sind nicht im DUDENUniversalwörterbuch (2001) aufgenommen, vgl. dazu Couper-Kuhlen/Reber 2010).

ach, ah, aha, au(a)/autsch, bäh, brrr, hm, hihi, hoppla, hu(ch), hui, hurra, igitt, ih*, juhu, na, naja*, nanu, oh, oho, oi ${ }^{*}$, oje, pah, pfui, phh*, puh, tja, uff, uh, ui

Wir finden auch noch die folgenden weitgehend konventionalisiert und in diese Liste gehörend: ätsch, he/hej, pst/pscht. Die Schreibung der Interjektionen ist nicht normiert, aber doch weitgehend konventionalisiert. 


\section{Tonhöhenbewegung und Funktion}

Bei einer Reihe von Interjektionen ist es die Tonhöhenbewegung, die den entscheidenden Beitrag zu ihrer Funktion im jeweiligen Kontext liefert; oder anders ausgedrückt: Die Tonhöhenbewegung »hat hier phonemischen Wert wie in Tonsprachen « (Schwitalla ${ }^{3}$ 2006: 157). Interessant für die Prosodie sind die Interjektionen, die mehr als eine Tonhöhenbewegung haben können. Das sind vor allem ach, ah, aha, hm, na, oh und oho. Bei diesen Interjektionen

»liegt tendenziell >Prosodie pur`vor, weil sie weitgehend frei von segmentell-lexikalischen $[\ldots]$ und grammatischen Informationen sind « (Kehrein 2002: 67).

Interjektionen »verweisen also auf nichts in der Welt « (Schwitalla 1990: 174). Ihre jeweilige Bedeutung bekommen sie erst durch das prosodische Mittel Tonhöhen- bewegung. Leicht überspitzt kann man sagen: Die Tonhöhenbewegung ist ihre Bedeutung. Diese Unterklasse der Interjektionen ist damit das einzige tonsprachliche Element des Deutschen (vgl. Ehlich 1986: 54). Bei diesen Interjektionen trifft also auf schöne Weise der berühmte Satz von Wittgenstein aus den Philosophischen Untersuchungen zu (den auch Ehlich 1986: 242 anführt): »Die Bedeutung eines Wortes ist sein Gebrauch in der Sprache." Ehlich (1986: 75) etabliert fünf Tonstrukturen, die potenziell vorkommen können:

gleich bleibend; steigend; fallend; steigend-fallend; fallend-steigend

Dazu gibt es oft noch kurze und reduplizierte Formen (hmhm, igittigitt). Am Vollständigsten sind die o. e. fünf Tonstrukturen und die damit verbundenen »Bedeutungen « bei $h m$ ausgebildet:

\begin{tabular}{||l|l||}
\hline \hline & Zögern, Nachdenken, Zweifel; vielleicht, aber, ich weiß nicht \\
\hline$\backslash$ & Widerspruch, unterschiedliche Meinung; wieso das denn?, was sagst du da? \\
\hline$\wedge$ & Ratlosigkeit, Überraschung; das ist ja merkwürdig, da haben wir den Salat! \\
\hline$\vee$ & Zustimmung; einverstanden, okay \\
\hline$\vee$ & Bewunderung, Lob, angenehmes Gefühl; lecker, das ist prima \\
\hline \hline
\end{tabular}

Interjektionen im Unterricht

In dem Maße, in dem die Lehrwerkdialoge in den letzten 20 Jahren natürlicher und damit authentischer geworden sind (vgl. Vorderwülbecke 2008: 278), tauchen in den Lehrwerkdialogen zunehmend Interjektionen auf. Sie werden aber meist nicht als relevante Handlungseinheiten bewusst gemacht und geübt, sondern rein lexikalisch betrachtet.

»Im Gegensatz zu den anderen in den Dialogen eingeführten lexikalischen und grammatischen Phänomenen werden aber Funktion und Bedeutung der Interjektionen in den jeweiligen Lehrwerken nicht erklärt [...]«. (Schmidt 2004: 106)

Wenn im Unterricht vermittelt werden kann, dass und wie die jeweils konkret beabsichtigte Funktion einer Interjektion von der Tonhöhenbewegung abhängt, kann generell der große Einfluss prosodischer Mittel auf die Gestaltung verbaler Interaktion deutlich werden, z. B. bei der Markierung sozialer Beziehungen oder dem Ausdruck von Gefühlen. All das sind Elemente von Bedeutung, die für das Verstehen hoch relevant sind. So könnte das Erreichen des Lernziels kommunikative Kompetenz in einem weiteren Sinne befördert werden.

Man kann zunächst am Beispiel von $H M$ die fünf Tonhöhenbewegungen in einer Tabelle vorgeben und diesen Tonkonturen jeweils (möglichst vom Tonträger) 
gehörte HM-Varianten zuordnen. Weiterhin kann man HM mit einer bestimmten Tonhöhenbewegung aus mehreren prosodischen Varianten heraushören lassen. Erst nach einer Reihe solcher Diskriminations- und Identifikationsübungen sollte man - immer im Kontext eines Ausspruchs - hören und nachsprechen lassen. Auch im Rahmen von Dialogarbeit können Interjektionsübungen sinnvoll eingebaut werden.

Sozusagen als Kür (und eher auf höheren Lernstufen) können die Lerner schließlich selber Empfindungen oder Einstellungen zum Gesagten mithilfe von Interjektionen formulieren, die sie dann auch mit der intendierten Tonkontur laut vortragen sollten

\section{Ausblick}

Wir haben einige aus unserer stärker phonologisch geprägten Sicht wichtige und vernachlässigte Themen aus dem Bereich Prosodie vorgestellt und jeweils auch einige Anregungen für die Praxis angeboten. Es wäre sicherlich sinnvoll, solche Anregungen in größerem Umfang zu sammeln und weiterzugeben, damit diese prosodischen Mittel aus ihrem didaktischen Dornröschenschlaf geweckt werden können.

Obwohl die übergeordnete Bedeutung der Prosodie für die »Ausspracheschulung « weitgehend anerkannt ist, gibt es also aufgrund der erwähnten Defizite noch viel zu tun, was eine Didaktik und Methodik der Prosodie beschreiben und vermitteln müsste. Vor allem sollten auch Ergebnisse neuerer phonologischer Prosodieforschungen mit einbezogen werden.

Diese neue Didaktik muss zum einen dazu beitragen, prosodische Prozesse verständlich $\mathrm{zu}$ machen, zum anderen aber auch Wissen und praktische Anregungen für die Unterrichtsarbeit geben.
So wären z. B. zur Orientierung für die Lehrenden Überblickstabellen nützlich, in denen die prosodischen Hauptprobleme des Deutschen für Sprecher von möglichst vielen Ausgangssprachen aufgezeigt werden. Das könnte eine hilfreiche Ergänzung bzw. Erweiterung zu der (stark auf Einzellaute fokussierten) Beschreibung phonetischer Interferenzen zwischen dem Deutschen und 30 weiteren Sprachen sein, die Dieling (1992) vorgelegt hat.

Alle Bemühungen um die Phonetik im DaF-Unterricht könnte man unter dem Ziel des Aufbaus einer »akustischen Intelligenz« zusammenfassen (wie Daniel Barenboim das für die musikalische Erziehung genannt hat). Für die Belange des hier behandelten Themas könnte das Ziel einer »prosodischen Intelligenz « formuliert werden.

\section{Literatur}

(Wir haben die im Text erwähnten Lehrwerke als bekannt vorausgesetzt und sie deshalb nicht im Literaturverzeichnis aufgenommen).

Auer, Peter; Selting, Margaret: »Der Beitrag der Prosodie zur Gesprächsorganisation«. In: Brinker, Klaus; Heinemann, Wolfgang; Sager, Sven F. (Hrsg.): Textund Gesprächslinguistik. Ein internationales Handbuch zeitgenössischer Forschung. 2. Hlbbd. »Gesprächslinguistik«. Berlin; New York: de Gruyter, 2001, 1122-1131 (Handbücher zur Sprach- und Kommunikationswissenschaft, HSK 16.2).

Bertinetto, Pier Marco: Strutture prosodiche dell'italiano (Prosodische Strukturen des Italienischen). Firenze: Accademia della Crusca, 1981 (Studi di grammatica italiana).

Bose, Ines: »Rhythmus im Unterricht Deutsch als Fremdsprache. Einige Überlegungen zu aktuellen Tendenzen in Phonetiklehrwerken ", Deutsch als Fremdsprache 36, 4 (1999), 225-229.

Büring, Daniel: »Intonation und Informationsstruktur «. In: Blühdorn, Hardarik; Breindl, Eva; Waßner, Ulrich Hermann 
(Hrsg.): Text - Verstehen. Grammatik und darüber hinaus. Berlin; New York: de Gruyter 2006, 144-163 (Jahrbuch des Instituts für deutsche Sprache 2005).

Couper-Kuhlen, Elizabeth; Reber, Elisabeth: »Interjektionen zwischen Lexikon und Vokalität: Lexem oder Lautgestalt?« In: Deppermann, Arnulf; Linke, Angelika (Hrsg.): Sprache intermedial. Stimme und Schrift, Bild und Ton. Berlin: de Gruyter, 2010 (in Druck) (Jahrbuch des Instituts für Deutsche Sprache 2009).

Dieling, Helga: Phonetik im Fremdsprachenunterricht Deutsch. Berlin; München; Wien: Langenscheidt, 1992.

Duden. Die Grammatik. 7. völlig neu erarbeitete und erweiterte Auflage. Hrsg. von der Dudenredaktion. Mannheim; Leipzig; Wien; Zürich: Dudenverlag, 2005 (Duden, 4).

Duden. Drosdowski, Günther; Alsleben, Brigitte (Hrsg.): DUDEN. Deutsches Universalwörterbuch $A-Z$. 4., neu bearbeitete und erweiterte Auflage. Mannheim; Wien; Zürich: Dudenverlag, 2001.

Ehlich, Konrad: Interjektionen. Tübingen: Niemeyer, 1986 (Linguistische Arbeiten, 111).

Féry, Caroline (2009): Der SFB 632: Informationsstruktur. http://www.sfb632. uni-potsdam.de/main3322.html.

Féry, Caroline: German Intonational Patterns. Tübingen: Niemeyer, 1993.

Fiehler, Reinhard: »Gesprochene Sprache«. In: Die Grammatik. 7. völlig neu erarbeitete und erweiterte Auflage. Hrsg. v. der Dudenredaktion. Mannheim; Leipzig; Wien; Zürich: Dudenverlag, 2005, 11751256 (Duden 4).

Fiehler, Reinhard: Kommunikation und Emotion. Theoretische und empirische Untersuchungen zur Rolle von Emotionen in der verbalen Interaktion. Berlin; New York: de Gruyter, 1990 (Grundlagen der Kommunikation und Kognition /Foundations of Communication and Cognition).

Fischer, Andreas: Deutsch lernen mit Rhythmus. Der Sprachrhythmus als Basis einer integrierten Phonetik im Unterricht Deutsch als Fremdsprache. Methode und Material. Leipzig: Schubert, 2007.

Graffmann, Heinrich; Moroni, Manuela: »Wie hören die Sätze auf? Tonbewegungen am Satzende und ihre Bedeutung für den Sprachunterricht «, Journal for foreign languages 3. http://www.gfl-journal.de/32007/graffmann_moroni.html.

Günthner, Susanne: »The prosodic contextualization of moral work: an analysis of reproaches in >why<-formats «. In: Couper-Kuhlen, Elizabeth; Selting, Margaret (Hrsg.): Prosody in Conversation. Interactional Studies. Cambridge: Cambridge University Press, 1996, 271-302.

Heidolph, Karl-Erich; Flämig, Walter; Motsch, Wolfgang: Grundzüge einer deutschen Grammatik. Berlin: Akademie-Verlag, 1981.

Henrici, Gert; Riemer, Claudia (mit Arbeitsgruppe) (Hrsg.): Einführung in die Didaktik des Unterrichts Deutsch als Fremdsprache mit Videobeispielen. 2 Bände. Baltmannsweiler: Schneider Hohengehren, 1994.

Höhle, Tilman: »VERUM-Fokus«, Sprache und Pragmatik 5 (1988), 1-7.

Huneke, Hans-Werner; Steinig, Wolfgang: Deutsch als Fremdsprache. Eine Einführung. Berlin: Erich Schmidt 1997, ${ }^{3} 2002$ (Grundlagen der Germanistik, 34).

Kehrein, Roland: Prosodie und Emotionen. Tübingen: Niemeyer, 2002.

Lerner, Jean-Yves: »Bedeutung und Struktursensitivität der Modalpartikel doch ", Linguistische Berichte 109 (1987), 203-229.

Lötscher, Andreas: Satzakzent und Funktionale Satzperspektive im Deutschen. Tübingen: Niemeyer, 1983 (Linguistische Arbeiten, 127).

Missaglia, Federica: Studi sul bilinguismo scolastico italo-tedesco. Brescia: editrice La Scuola, 1997 (Studien zum Bilingualismus bei Lernern italienisch-deutsch).

Moroni, Manuela: »Zur Signalfunktion der Modalpartikeln für die Prosodie«. In: Abel, Andrea; Stuflesser, Mathias; Putz, Magdalena (Hrsg.): Mehrsprachigkeit in Europa: Erfahrungen, Bedürfnisse, Gute Praxis. Tagungsband. - Plurilinguismo in Europa: esperienze, esigenze, buone pratiche. Atti del convegno. - Multilingualism across Europe: Findings, Needs, Best Practices. Proceedings. 24.-26.08.2006, Bolzano/Bozen. Bozen: Eurac, 2006, 381-390.

Nübling, Damaris: »Die prototypische Interjektion: Ein Definitionsvorschlag «, Zeitschrift für Semiotik 1-2 (2004), 11-46.

Oppenrieder, Wilhelm: »Intonatorische Kennzeichnung von Satzmodi«. In: Altmann, Hans (Hrsg.): Intonationsforschungen. Tübingen: Niemeyer, 1988, 169-205. 
Peters, Jörg: »Intonation«. In: Duden. Die Grammatik. 7. völlig neu erarbeitete und erweiterte Auflage. Hrsg. v. der Dudenredaktion. Mannheim; Leipzig; Wien; Zürich: Dudenverlag, 2005, 95-128 (Duden, 4).

Reinke, Kerstin: »Zur emotionalen Wirkung phonetischer Mittel bei DaF-Lernenden «, Zeitschrift für interkulturellen Fremdsprachenunterricht 12, 2 (2007), 37 S. (http:// spz1.spz.tu-darmstadt.de/projekt_ejournal/jg-12-2/docs/Reinke.pdf.

Rösler, Dietmar: Deutsch als Fremdsprache. Stuttgart; Weimar: Metzler, 1994.

Scherer, Klaus: Vokale Kommunikation - Nonverbale Aspekte des Sprachverhaltens. Weinheim: Beltz, 1982.

Scherer, Klaus; Wallbott, Harald: »Ausdruck von Emotionen «. In: Scherer, Klaus (Hrsg.): Psychologie der Emotionen. Göttingen: Hogrefe, 1990 (Enzyklopädie der Psychologie: Motivation und Emotion, Bd. 3).

Schmidt, Claudia: »Interjektionen im Zweitsprachenerwerb «, Zeitschrift für Semiotik 26 (2004), 11-46.

Schwerdtfeger, Inge Christine: »Auf der Suche nach den verlorenen Emotionen «, Info DaF 24, 5 (1997), 587-606.

Schwitalla, Johannes: Gesprochenes Deutsch. Eine Einführung. Berlin: Erich Schmidt, 1. Auflage 1997, 3. Auflage 2006.

Selting, Margret u.a.: »Gesprächsanalytisches Transkriptionssystem (GAT) «, Linguistische Berichte 173 (1998), 91-122.

Selting, Margret: Prosodie im Gespräch. Aspekte einer interaktionalen Phonologie der Konversation. Tübingen: Niemeyer, 1995 (Linguistische Arbeiten, 329).

Stock, Eberhard: Deutsche Intonation. Berlin; München; Leipzig: Langenscheidt, 1996.

Stock, Eberhard; Hirschfeld, Ursula (Hrsg.): Phonothek Deutsch als Fremdsprache. Berlin; München; Leipzig: Langenscheidt, 1996.

Storch, Günther: Deutsch als Fremdsprache Eine Didaktik. Theoretische Grundlagen und praktische Unterrichtsgestaltung. München: Fink, 1999 (UTB L).

Tischer, Bernd: Die vokale Kommunikation von Gefühlen. Weinheim: Beltz, 1993.

Uhmann, Susanne: Fokusphonologie. Tübingen: Niemeyer, 1991.
Veličkova, Ljudmilla: »Der Rhythmus im Deutschen «, Deutsch als Fremdsprache 36, 4 (1999), 220-224.

Vorderwülbecke, Klaus: »Sprache kommt von Sprechen - Gesprochene Sprache im DaF-Unterricht«. In: Chlosta, Christof; Leder, Gabriele; Krischer, Barbara (Hrsg.): Auf neuen Wegen. Deutsch als Fremdsprache in Forschung und Praxis. Göttingen: Universitätsverlag, 2008, 261-278 (Materialien Deutsch als Fremdsprache, 79).

Vorderwülbecke, Klaus: »Da ist Musik drin - Prosodie, gesprochene Sprache und Grammatik«. In: Breindl; Eva; Gunkel, Lutz; Strecker, Bruno (Hrsg.): Grammatische Untersuchungen, Analysen und Reflexionen. Festschrift für Gisela Zifonun. Tübingen: Narr, 2006, 567-580 (Studien zur deutschen Sprache, 36).

Zifonun, Gisela; Hoffmann, Ludger; Strekker, Bruno u. a.: Grammatik der deutschen Sprache. 3 Bde. Berlin; New York: de Gruyter, 1997.

\section{Manuela Moroni}

Studium der Germanistik und Anglistik an den Universitäten Bergamo und Saarbrücken; 2006 Promotion im Fach Allgemeine Sprachwissenschaft an der Universität Verona mit einer Dissertation zur Syntax der deutschen Modalpartikeln in Relation zur Prosodie und Informationsstruktur; 2004 bis 2007 Gastwissenschaftlerin in der Abteilung Grammatik des IDS in Mannheim; Dozentin für germanistische Linguistik an den Universitäten Mannheim und Bergamo; seit November 2007 wissenschaftliche Mitarbeiterin für Germanistische Linguistik an der Universität Trient; Hauptforschungsgebiete: Modalpartikeln, Informationsstruktur, Prosodie.

\section{Heinrich Graffmann}

Studium der Germanistik und Romanistik in Marburg, Paris, Grenoble, Heidelberg; nach einer Phase als Deutschlehrer und Französischlehrer Arbeit in Institutionen 
der Lehrerausbildung- und Lehrerweiterbildung in Deutschland, Argentinien, Brasilien; Arbeitsgebiete: Didaktik Deutsch als Fremdsprache mit Schwerpunkt auf Grammatik und Phonetik; zur Zeit Arbeit am Instituto de Enseñanza Superior en Lenguas Vivas »Juan Ramón Fernández « in Buenos Aires. Veröffentlichungen unter www.heinrich-graffmann.de

Klaus Vorderwülbecke

Dr. h. c.; Studium der Anglistik, Geschichte und Pädagogik an den Universitäten Bonn, Aix-en-Provence, Wien und
Washington State University, USA; nach dem Studium vier Jahre DAAD-Lektor an der Hokkaido-Universität in Sapporo, Japan. Von 1973 bis 2005 wissenschaftlicher Mitarbeiter am Institut für Deutsche Sprache, Mannheim; 1993-97 beurlaubt für die Mitarbeit an dem Fernsehsprachkurs »Einblicke« von Goethe-Institut und Inter Nationes; Mitautor mehrerer DaFLehr-Lernmaterialien; Fortbildungsseminare für DaF-Lehrende im In- und Ausland; thematische DaF-Schwerpunkte: Lehrwerkanalyse, Phonetik, Landeskunde. 